\title{
Blood pressure and heart rate variability and baroreflex sensitivity before and after brain death
}

\author{
F Conci, M Di Rienzo, P Castiglioni
}

\begin{abstract}
Objectives-To evaluate spontaneous blood pressure and heart rate variability and spontaneous baroreflex sensitivity before and after brain death.

Methods-Spontaneous variability of arterial blood pressure and heart rateestimated by power spectra of systolic (SBP) and diastolic blood pressure (DBP) and pulse interval (PI)-and spontaneous baroreflex sensitivity (BRS)-estimated by the alpha index and the sequence technique-were evaluated in 11 patients twice: shortly before and 1 hour after the onset of brain death.

Results-Significant spectral changes occurred after brain death: a general power reduction in PI spectra; a shift of SBP, DBP and PI powers toward the lower frequencies, resulting in a greater slope of the " $1 / f$ " spectral trends; and a marked reduction of SBP and DBP powers (-93\%) and of SBP-PI coherence $(-63 \%)$ at 0.1 Hz. The estimated average BRS was relatively high before brain death (around 11 $\mathrm{ms} / \mathrm{mm} \mathrm{Hg}$ ), and fell close to 0 or even was not detectable at all after brain death.

Conclusions-Parameters describing spontaneous blood pressure and heart rate variability and indexes reflecting the baroreflex function, which were relatively normal up to a few hours before brain death, underwent marked changes with the onset of brain death. All the changes found are likely to reflect the cessation of activity of the cardiovascular brain stem centres. These findings indicate that techniques of blood pressure and heart rate spectral analysis and of dynamic assessment of baroreflex sensitivity may be useful to complement the diagnosis of brain stem death.
\end{abstract}

(F Neurol Neurosurg Psychiatry 2001;71:621-631)

Keywords: power spectrum; computer assisted diagnosis; brain stem death

Brain stem death is a fundamental requisite in the two principal definitions of brain death. Indeed, brain death is defined as "irreversible cessation of all functions of the entire brain, including the brain stem" in the United States, ${ }^{1}$ and as "permanent functional death of the brain stem" in the United Kingdom. ${ }^{2}$ Currently, the diagnosis of brain stem death is assessed by checking the absence of consciousness, spontaneous postures, and breathing, and the irreversible loss of specific brain stem reflexes-pupillary light, corneal, oculocephalic, oculovestibular, oropharyngeal, and cough reflexes. ${ }^{12}$

Among the criteria for determining brain death, the assessment of a permanent loss of cardiovascular regulation has never been considered, although major alterations in cardiovascular dynamics can be expected from the impairment of the cardiovascular centres located in the brain stem. Actually, changes in heart rate and arterial blood pressure variability, and in the baroreflex function have been seen in brain dead patients, when compared with vegetative patients or healthy controls. ${ }^{3-10}$ These data, however, have invariably been collected by comparing stable brain dead patients against external groups of control subjects. Thus, no information is currently available on the process of decay, within the same subject, of the functionality of cardiovascular centres from the state of impending brain death to the complete inactivity of the brain stem. In particular, it is still unknown whether substantial modifications in the activity of cardiovascular brain stem centres coincide with the brain stem death, or they already appear before the death, given the critical conditions of these patients. From a methodological perspective, such knowledge could contribute to clarify whether the analysis of cardiovascular dynamics may be used as an additional tool for determining brain death.

In the present study we considered this unexplored issue by evaluating the blood pressure and heart rate variability and the sensitivity of baroreflex control of the heart in a group of comatose patients before and after brain stem death.

For the evaluation of variability we made use of the spectral analysis. This approach allowed us to separately quantify the power (a quantity related to the amplitude) of each fluctuation forming blood pressure and heart rate variability. In this way, we could focus separately on fluctuations synchronous with respiration, on the so called " 10 seconds rhythm", and on fluctuations with periods longer than 10 seconds. This was done on the basis of previous evidence suggesting that (1) at the respiratory frequency, heart rate fluctuations reflect the vagal control of the heart whereas fluctuations in blood pressure reflect the mechanical coupling between respiration and haemodynamics; (2) heart rate fluctuations with period of about 10 seconds reflect both the sympathetic and parasympathetic control of the heart whereas oscillations in blood pressure with the same period may be, at least in part, under the baroreflex influence; and (3) fluctuations in 
blood pressure and heart rate with periods longer than 10 seconds, although not associated with a specific biological mechanism, have been shown to convey important physiological information. ${ }^{112}$ In particular, the spectral power of these slower fluctuations tends to be inversely related to the frequency according to a $1 / f^{\beta}$ law, and the coefficient $\beta$ of this relation was found to have a marked prognostic value. ${ }^{13}$

Moreover, the effects of brain stem death on the baroreflex control of the heart were investigated in our study by using recent techniques based on the evaluation of spontaneous changes in blood pressure and heart rate beat to beat variability. At variance from traditional approaches for the evaluation of baroreflex sensitivity, these techniques do not require any specific stimulation of the patient and allowed us to obtain an almost continuous quantification of the baroreflex sensitivity without introducing any disturbance to the patient, or interfering with his or her clinical care.

\section{Methods}

The study was approved by the ethics committee of the Niguarda Hospital in Milan, Italy. Eleven patients (six men; five women) aged between 21 and 66 years (mean age 49 years) admitted to hospital in the neurological intensive care unit were enrolled in the study. The causes of admission to hospital were head injury, subarachnoid haemorrhage, cerebral haemorrhage, and cerebral tumours (table 1). At the time of admission no patient had a medical history of neurological and cardiovascular disorders. All patients were in a coma, with a Glasgow coma scale of 3 to 4 . Evaluation by computed axial tomography showed irreversible lesions not susceptible to pharmacological or surgical interventions, and their prognosis was negative in a very short time. Patients received standard procedures for monitoring arterial blood pressure and ECG, and standard therapy to maintain physiological homeostasis. They were mechanically ventilated, and a gasping respiratory activity was present in all patients before brain death. Four patients (Nos 5, 6, 8, and 11 in table 1) received catecholamines before brain death to improve systemic pressure and intracerebral perfusion pressure (dobutamine or dopamine $<10 \mu \mathrm{g} / \mathrm{kg} / \mathrm{h}$ and/or noradrenaline (norepinephrine) $<0.1 \mu \mathrm{g} / \mathrm{kg} / \mathrm{h}$ ). All patients but one (No 10) received catecholamines after brain death to maintain systolic blood pressure greater than $100 \mathrm{~mm} \mathrm{Hg}$ (dopamine $<10 \mu \mathrm{g} / \mathrm{kg} / \mathrm{h}$ and/or noradrenaline $<0.1 \mu \mathrm{g} / \mathrm{kg} / \mathrm{h})$. In each patient it has been verified that infusion pumps did not produce any fluctuation in the arterial blood pressure due to intermittent infusion.

Intra-arterial blood pressure was measured by a catheter inserted into the radial artery. Blood pressure was recorded continuously from the time of admission up to the establishment of brain death and during the following legal observation period ( 6 hours for adults) after which the patient could be moved to the operating theatre for possible organ donation. Brain death was identified in all patients according to the Italian legal criteria for adults. These criteria
Table 1 Clinical features of patients

\begin{tabular}{llll}
\hline No & Age $(y)$ & Sex & Cause of admission to hospital \\
\hline 1 & 21 & F & Head injury \\
2 & 52 & M & Subarachnoid haemorrhage \\
3 & 58 & M & Cerebral haemorrhage \\
4 & 66 & F & Subarachnoid haemorrhage \\
5 & 40 & F & Head injury \\
6 & 51 & F & Subarachnoid haemorrhage \\
7 & 58 & M & Cerebral haemorrhage \\
8 & 59 & M & Head injury \\
9 & 61 & F & Cerebral tumour \\
10 & 41 & M & Subarachnoid haemorrhage \\
11 & 35 & M & Head injury \\
\hline
\end{tabular}

required verification of apnoea at $\mathrm{pCO}_{2}>60 \mathrm{~mm}$ $\mathrm{Hg}$, absence of brain stem reflexes for a period of 6 hours, with no hypothermia or drug induced depression, and recordings of isoelectric EEG for periods of 30 minutes every 2 hours during the observation period.

Twelve hour blood pressure recordings, each including about 6 hours before and 6 hours after brain death were considered for the study. Arterial blood pressure was sampled at $125 \mathrm{~Hz}$ and edited by an interactive procedure to eliminate possible artefacts. Systolic and diastolic blood pressures (SBP and DBP) were identified on a beat to beat basis and the cardiac rhythm was derived from the blood pressure wave by computing the pulse interval (PI), defined as the time interval between consecutive systolic peaks. Two segments of data, one before and one after brain death, were identified for the subsequent analyses. These segments-hereafter defined "before BD" and "after BD" respectively-were selected so as not to include vegetative storms or neurogenic spinal shocks possibly occurring close to the onset of brain death (fig $1 \mathrm{~A}-\mathrm{B}$ ). The reason for the exclusion is that these events are characterised by sudden and pronounced blood pressure rises and falls, ${ }^{14-16}$ and such a circulatory instability might interfere with the data analysis and be a confounding factor in the final interpretation of the results. On average, the before BD and after BD segments lasted 3 and 4 hours respectively. The after $\mathrm{BD}$ segment started 1 hour after the onset of brain death.

ANALYSIS OF BLOOD PRESSURE AND HEART RATE VARIABILITY

As mentioned in the introduction, cardiovascular variability was evaluated by making use of the spectral analysis. Briefly, each SBP, $\mathrm{DBP}$, and PI series was split into short term data records, each lasting 512 seconds, and for each record the power spectrum was estimated by the fast Fourier transform. A typical output of this procedure is illustrated in figure 1, panels C and D. The spectral characteristics remained quite stable in the before $\mathrm{BD}$ and after BD segments, thus the spectra falling in each of these segments have been averaged to obtain a single spectrum for each condition. The respiratory component was easily identified in each spectrum by visual inspection, appearing as a clear and sharp spectral peak at frequencies higher than $0.1 \mathrm{~Hz}$. The magnitude of the "10 seconds rhythm" was quantified by integrating the spectrum between 0.06 
and $0.12 \mathrm{~Hz}$. The slowest components of variability have been globally quantified by estimating the $\beta$ exponent of the $1 / f^{\beta}$ law relating the power of heart rate or blood pressure spectra with the frequency $f$ at the lower frequencies of the spectrum. When both the vertical (power density) and horizontal (frequency) axes of a spectrum are represented in a $\log$ scale, the $1 / f^{\beta}$ trend is transformed into a linear trend with slope $-\beta$. Thus, once the spectrum was plotted in a log-log scale, the $\beta$ exponent was estimated by computing the slope of the regression line between power and frequency in the band ranging from $4 \times 10^{-3}$ to $2 \times 10^{-2} \mathrm{~Hz}^{11}{ }^{13}$
The degree of coupling between SBP and PI was also assessed through the estimation of the squared coherence modulus $|\mathrm{k}(f)|^{2}$. Similarly to the squared correlation coefficient $r^{2}$, which quantifies the degree of linear correlation between two variables, the coherence modulus $|\mathrm{k}(f)|^{2}$ gives the degree of linear correlation between two signals-SBP and PI in this case-as a function of the frequency $f^{17}$ Similarly to $r^{2},|\mathrm{k}(f)|^{2}$ may also range between 0 (when the two signals are completely uncorrelated at the frequency $f$ ) and 1 (perfect linear coupling). As for spectral analysis, each data series was broken up in 512 second long data records, and $|\mathrm{k}(f)|^{2}$ was estimated before and
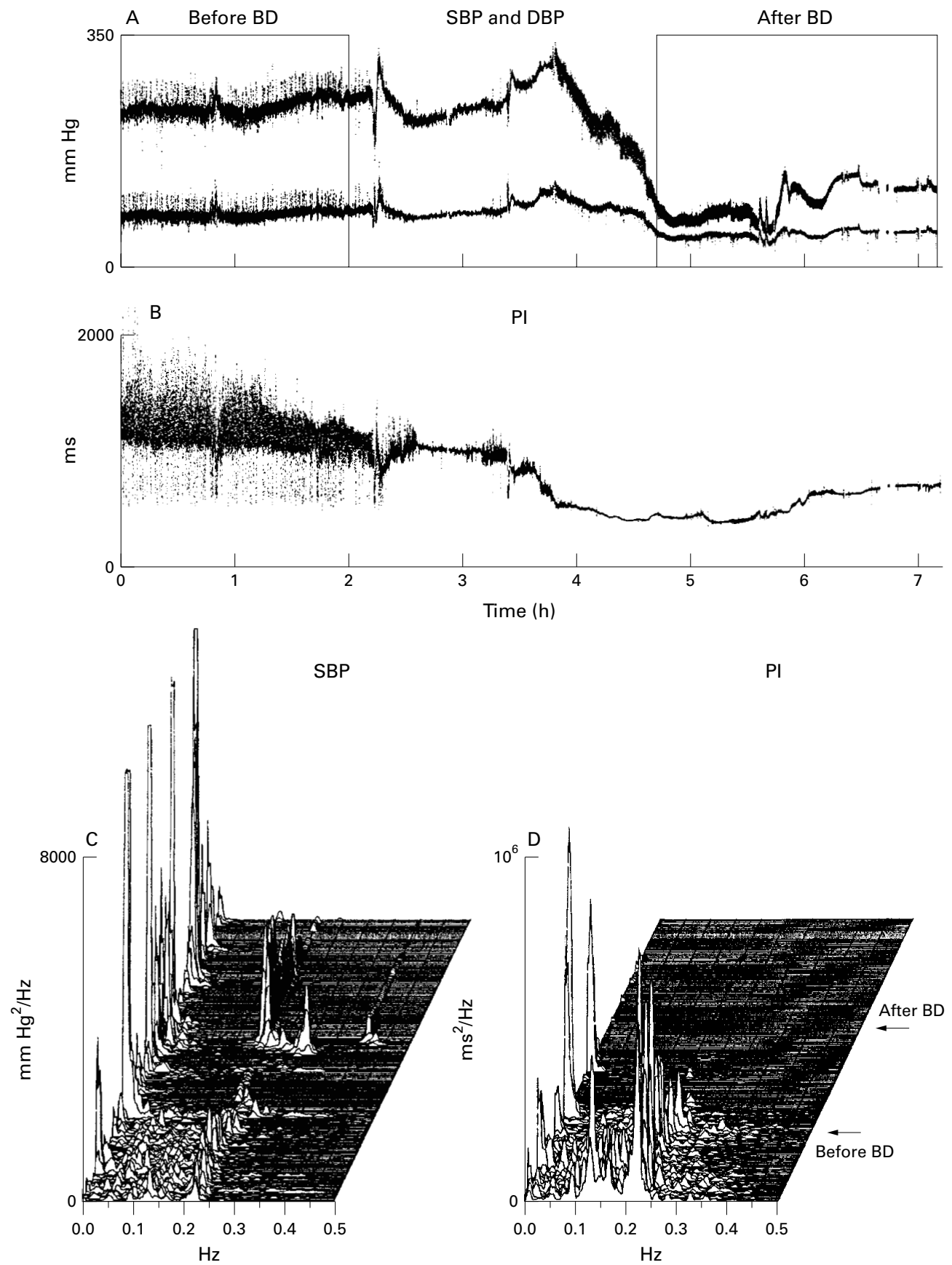

Figure 1 (A) Systolic and diastolic blood pressure; (B) pulse interval; (C) power spectra of systolic blood pressure; (D) power spectra of pulse interval; power spectra were computed sequentially over contiguous data segments in a representative subject (No 3 in table 1). Before and after brain death periods are indicated. $S B P=$ systolic blood pressure; $D B P=$ diastolic blood pressure; $P I=$ pulse interval; Before $B D=$ before brain death period; $A$ fter $B D=$ after brain death period. 
after brain death by averaging the estimates referring to data records falling in the before $\mathrm{BD}$ and after BD segments.
Further technical details on data preprocessing, and on the estimation of spectra and coherences are reported in the appendix.
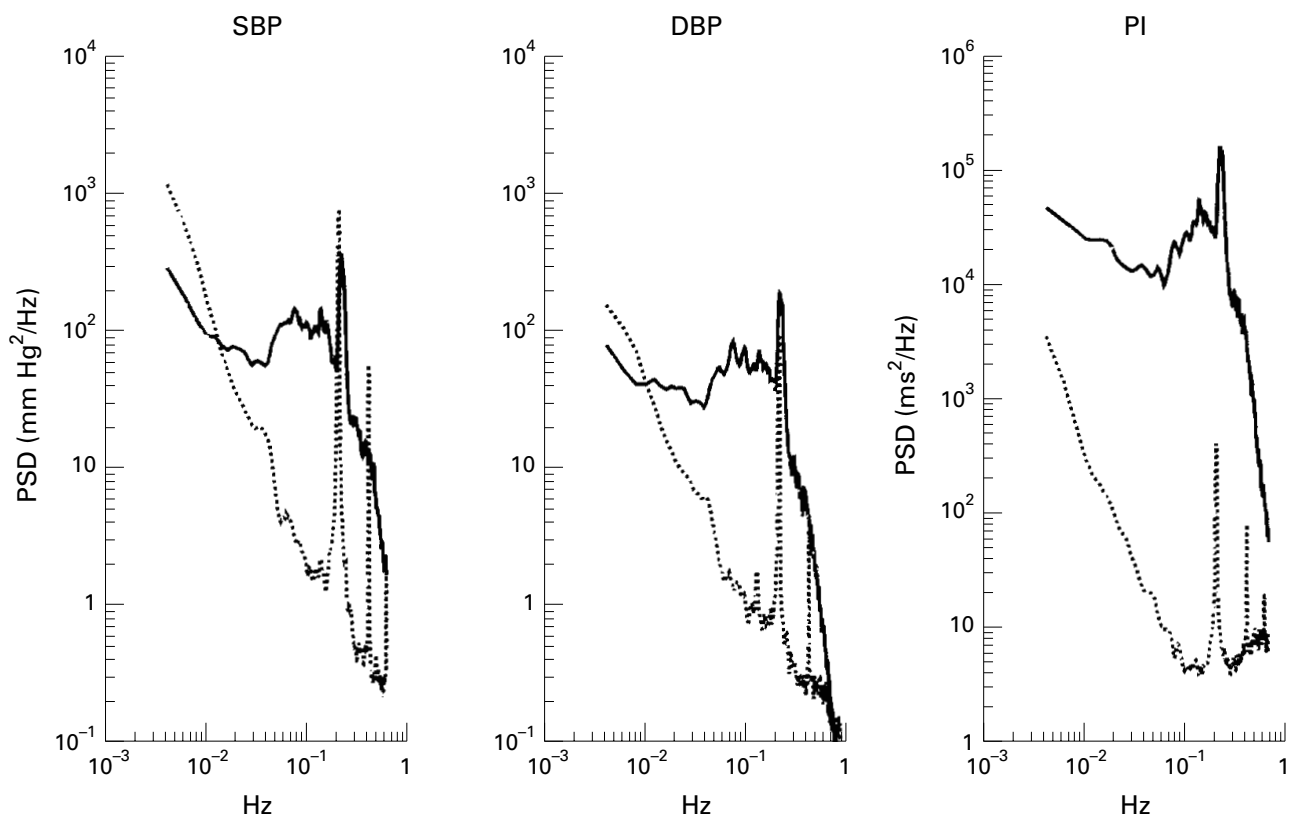

Figure $2 S B P, D B P$, and PI power spectral densities (PSD) in a representative patient (the same as in fig 1) before (continuous line) and after (dotted line) brain death. Because the spectra span over four decades of power and three decades of frequency, a log-log scale is used to facilitate the identification of spectral details. Abbreviations as in fig 1.
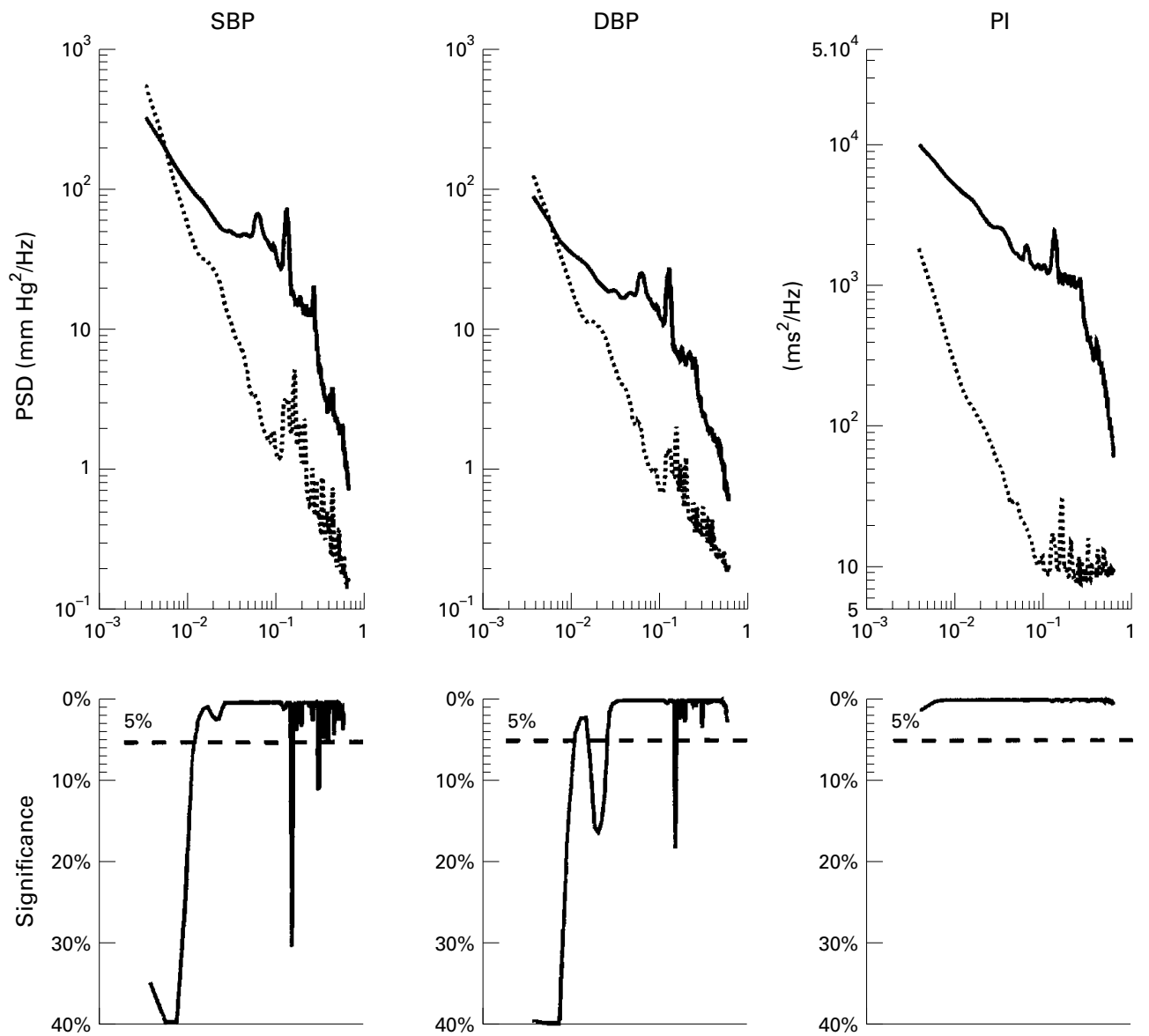

Figure 3 Spectral analysis. Upper panels: log-log spectra averaged over the group of 11 patients before (continuous line) and after (dotted line) brain death; abbreviations as in fig 1. Lower panels: significance $p$ of the difference before $v$ after brain death for each spectral line; when $p$ is above the 5\% threshold (dashed line) the corresponding spectral lines differ at a significance level of $p<0.05$. 
Table 2 Individual powers of systolic (SBP) and diastolic blood pressure (DBP) and pulse interval (PI) spectra in the band between 0.06 and $0.12 \mathrm{~Hz}$ before $(B)$ and after $(A)$ brain death

\begin{tabular}{|c|c|c|c|c|c|c|c|c|c|}
\hline \multirow[b]{2}{*}{ Patient No } & \multicolumn{3}{|l|}{$S B P$} & \multicolumn{3}{|l|}{$D B P$} & \multicolumn{3}{|l|}{$P I$} \\
\hline & $B\left(m m H^{2}\right)$ & $A\left(m m H^{2}\right)$ & $\Delta(\%)$ & $B\left(m m H^{2}\right)$ & $A\left(m m H^{2}\right)$ & $\Delta(\%)$ & $B\left(m s^{2}\right)$ & $A\left(m s^{2}\right)$ & $\Delta(\%))$ \\
\hline 1 & 5.55 & 0.45 & -92 & 10.42 & 0.16 & -98 & 1344.8 & 0.45 & -100 \\
\hline 2 & 1.66 & 0.19 & -89 & 0.44 & 0.06 & -86 & 93.2 & 0.42 & -100 \\
\hline 3 & 7.07 & 0.16 & -98 & 3.91 & 0.07 & -98 & 1464.9 & 0.42 & -100 \\
\hline 4 & 16.05 & 0.55 & -97 & 4.03 & 0.33 & -92 & 408.8 & 1.78 & -100 \\
\hline 5 & 1.13 & 0.14 & -88 & 0.23 & 0.04 & -83 & 12.4 & 0.90 & -93 \\
\hline 6 & 0.44 & 0.03 & -93 & 0.30 & 0.03 & -90 & 14.2 & 0.38 & -97 \\
\hline 7 & 1.60 & 0.09 & -95 & 0.36 & 0.03 & -92 & 150.9 & 2.36 & -98 \\
\hline 8 & 4.58 & 0.10 & -98 & 0.54 & 0.06 & -90 & 1.2 & 0.88 & -24 \\
\hline 9 & 1.04 & 0.37 & -64 & 0.80 & 0.07 & -92 & 103.0 & 3.49 & -97 \\
\hline 10 & 3.00 & 0.34 & -89 & 1.55 & 0.19 & -88 & 196.8 & 13.38 & -93 \\
\hline 11 & 8.60 & 0.10 & -99 & 2.26 & 0.07 & -97 & 96.3 & 0.08 & -100 \\
\hline \multicolumn{3}{|c|}{ Median } & -93 & & & -92 & & & -98 \\
\hline \multirow{2}{*}{\multicolumn{3}{|c|}{$\begin{array}{l}0.1 \text { percentile } \\
0.9 \text { percentile }\end{array}$}} & -98 & & & -98 & & & -100 \\
\hline & & & -88 & & & -86 & & & -98 \\
\hline
\end{tabular}

$\Delta=$ The $\%$ reduction after brain death; $\Delta=(\mathrm{A}-\mathrm{B}) / \mathrm{B}$ : median and $0.1-0.9$ percentiles of $\Delta$ are also reported.

ASSESSMENT OF BAROREFLEX FUNCTION

To obtain an almost continuous monitoring of the baroreflex function without any intervention on the patient, we estimated the baroreflex sensitivity on the heart (BRS) from the spontaneous fluctuations of SBP and PI. Each technique employing this approach, however, derives information on the baroreflex function by evaluating the baroreflex response to a specific pattern of spontaneous blood pressure variability (usually represented by short blood pressure transients or rhythmic oscillations). Thus, given the different perspective from which each technique quantifies the baroreflex function, minor differences in the BRS estimates obtained by the various techniques can be expected, although it has been previously shown that when the BRS estimates are averaged over several minutes the differences tend to vanish in most instances. ${ }^{18}$ In this study we preferred to simultaneously estimate BRS by two independent and complementary methods to obtain a more comprehensive evaluation of the baroreflex function: the sequence technique, which focuses on the baroreflex response to pressure transients, ${ }^{19}$ and the calculation of the so called "alpha index", which reflects the baroreflex response to rhythmic oscillations. ${ }^{20}$ Through the sequence technique the BRS estimate was obtained as follows. The SBP and PI series were scanned automatically in a search for spontaneous sequences of three or more consecutive heart beats in which a progressive increase in SBP was followed, with a one beat lag, by a progressive PI lengthening or, vice versa, in which a progressive SBP reduction was followed by a shortening in PI. The slope of the regression line between SBP and PI values forming each sequence was taken as an estimation of the baroreflex sensitivity on the heart. In addition to the baroreflex sensitivity, we also computed the hourly rate of occurrence of spontaneous

Table 3 Mean (SD) of the slope of the regression line fitting the spectrum in a log-log scale

\begin{tabular}{llll}
\hline & Before BD & After BD & p Value \\
\hline SBP & $-0.909(0.389)$ & $-1.781(0.440)$ & 0.0002 \\
DBP & $-0.754(0.366)$ & $-1.538(0.376)$ & 0.0006 \\
PI & $-0.742(0.584)$ & $-1.629(0.414)$ & 0.0028 \\
\hline
\end{tabular}

$p=$ Statistical significance of the difference before $v$ after brain dead (abbreviations as in table 1). sequences, which can be taken as a rough index of the activity level of the baroreflex. The second estimation of the baroreflex sensitivity was obtained by computing the alpha index. Briefly, each pair of SBP and PI beat by beat series was sequentially split into 120 second long segments. Spectra and cross spectrum were computed in each segment. The SBP and PI powers were obtained by integrating spectral lines with a squared coherence modulus greater than 0.5 in the $0.07-0.6 \mathrm{~Hz}$ band. The

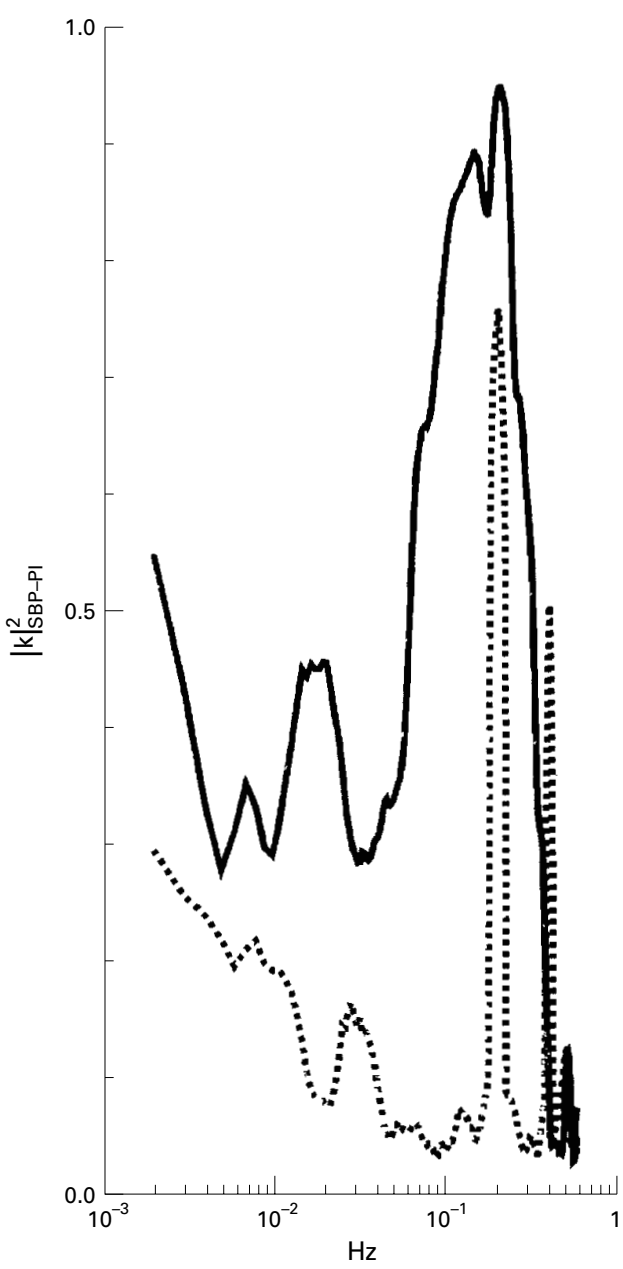

Figure 4 Squared coherence modulus between SBP and $P I,|k(f)|^{2}$ sp.pp in a representative patient (the same as in fig 1), before (continuous line) and after (dotted line) brain death. 

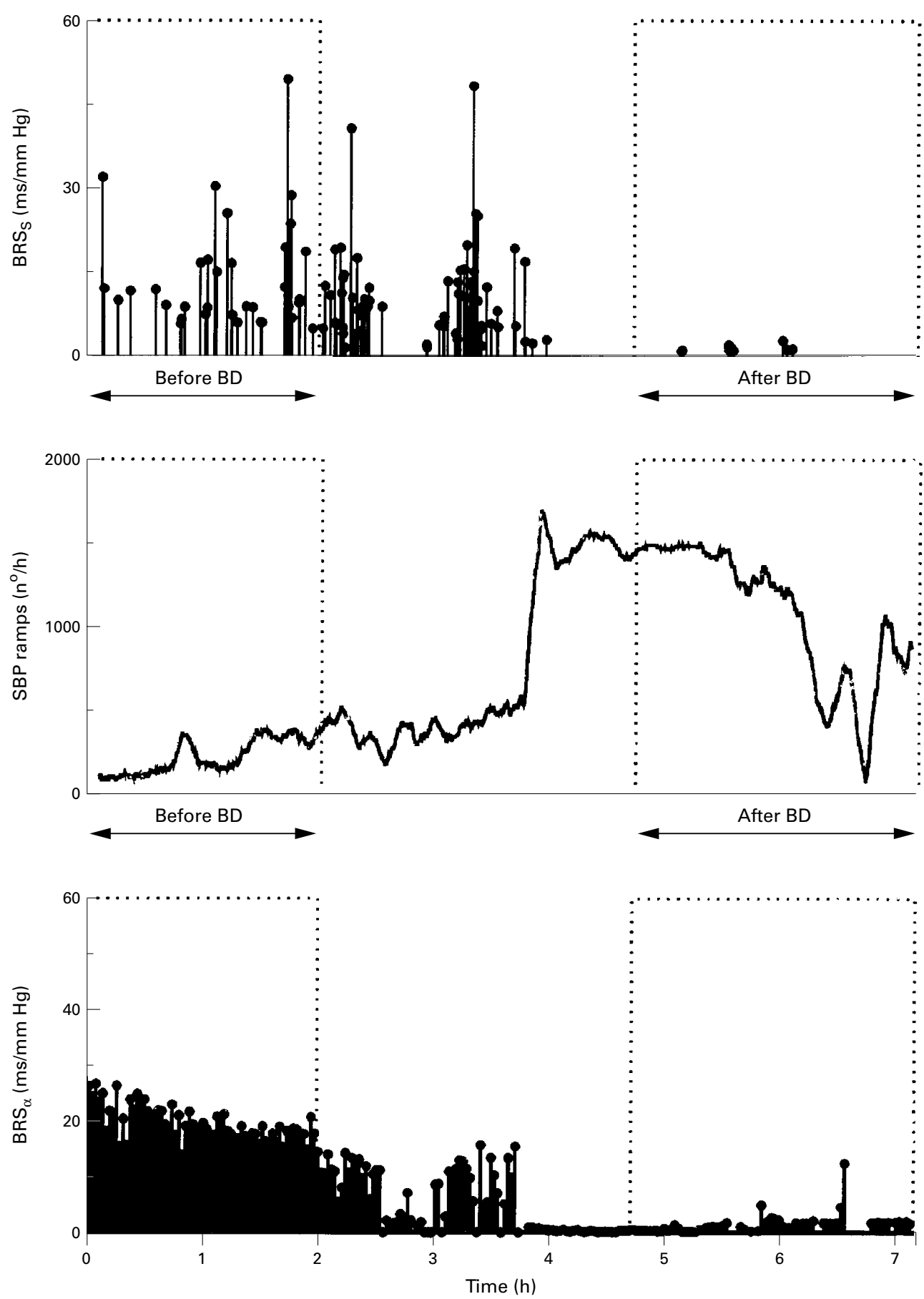

Figure 5 Dynamic assessment of spontaneous baroreflex activity in a representative patient (the same as in fig 1). From top to bottom: baroreflex sensitivity by the sequence technique $\left(B R S_{S}\right)$; rate of occurrence of systolic blood pressure ramps (No/hour); baroreflex sensitivity by the alpha index $\left(B R S_{a}\right)$.

root squared ratio between PI and SBP powers was taken as an estimate of the baroreflex sensitivity on the heart.

STATISTICAL ANALYSIS

For each quantity, the significance of the differences between the before $\mathrm{BD}$ and after $\mathrm{BD}$ segments was assessed by paired $t$ test. Log transformation of power spectra and archyperbolic-tangent transformation of coherence moduli were performed before statistical analysis to obtain normally distributed data. ${ }^{21}{ }^{22}$ The level of statistical significance was $p<5 \%$.

\section{Results}

Blood pressure and heart interval were lower after brain death. Ensemble average (mean (SD)) of SBP and DBP decreased from 175 (46) to 122 (23) $\mathrm{mm} \mathrm{Hg}$ and from 84 (17) to 68 (15) $\mathrm{mm} \mathrm{Hg}$ respectively; the heart interval decreased from 778 (208) ms to 586 (120) ms (all changes significant at $\mathrm{p}<0.05$ ).

ANALYSIS OF BLOOD PRESSURE AND HEART RATE VARIABILITIES

The spectra of a representative patient (No 3 in table 1) are shown in figure 2. Important 
changes were associated with brain death. The mechanical ventilator, set at 12 cycles/minute in this patient, produced a clear cut respiratory peak at $0.2 \mathrm{~Hz}$ in blood pressure and PI spectra. In the PI spectrum the amplitude of such a peak dropped by more than $99 \%$ (from 160000 to $400 \mathrm{~ms}^{2} / \mathrm{Hz}$ ) after brain death. The amplitude of the respiratory peak remained almost unchanged in the SBP and DBP spectra after death. In this case, however, it was the power of spectral components surrounding the respiratory peak which dramatically reduced. Brain stem death also resulted in a drastic power fall at $0.1 \mathrm{~Hz}$ and in a steepening of the " $1 / f$ " line representing the trend of the slowest components of variability in SBP, DBP, and PI spectra.

The spectra averaged over the whole group of patients and the level of statistical significance for the difference between before BD and after $\mathrm{BD}$ conditions are reported in figure 3. The results over the whole group reflected the findings obtained in the representative patient. Indeed, SBP, DBP, and PI powers at $0.1 \mathrm{~Hz}$ decreased in all patients after brain death, and the reduction was more than $90 \%$ in most of the cases, as shown in table 2. The slopes of the $1 / f$ lines became significantly steeper-more negative - after brain death, as also shown by the statistical analysis reported in table 3. Because of the greater slope, the power difference between before BD and after $\mathrm{BD}$ conditions progressively diminished ongoing towards the lower frequencies. For blood pressure, the difference became null at around $0.006 \mathrm{~Hz}$ and at lower frequencies the power was even greater (although not significantly) after brain death.

The average PI spectrum showed a significant and generalised power reduction at the respiratory frequency after brain death. By contrast, the SBP and DBP power reduction was limited to the region surrounding the respiratory frequency while at the very respiratory frequency no significant difference was found, as in the individual example of figure 2 . It is worth noting, however, that when individual spectra are mediated over the group, the averaging procedure tends to smear the respiratory peaks because these peaks occur at frequencies that vary from patient to patient, being the ventilator frequency tuned according to individual needs.

Figure 4 shows the degree of coupling between SBP and PI as quantified by the squared coherence modulus in the same patient of figures 1 and 2. Before brain death, the coherence modulus (and thus the degree of coupling) was remarkably high over a large frequency band, which includes the $0.1 \mathrm{~Hz}$ and the respiratory band. After brain death the coherence decreased consistently around 0.1 $\mathrm{Hz}$, but a narrow and high coherence peak remained at the respiratory frequency, indicating that a certain level of coupling between SBP and PI was still preserved. Similar results were found over the whole group of patients, and after brain death the average squared coherence dropped significantly at $0.1 \mathrm{~Hz}$ (from 0.54 to 0.16 ), preserving, however, a narrow respiratory peak centred at around 0.14

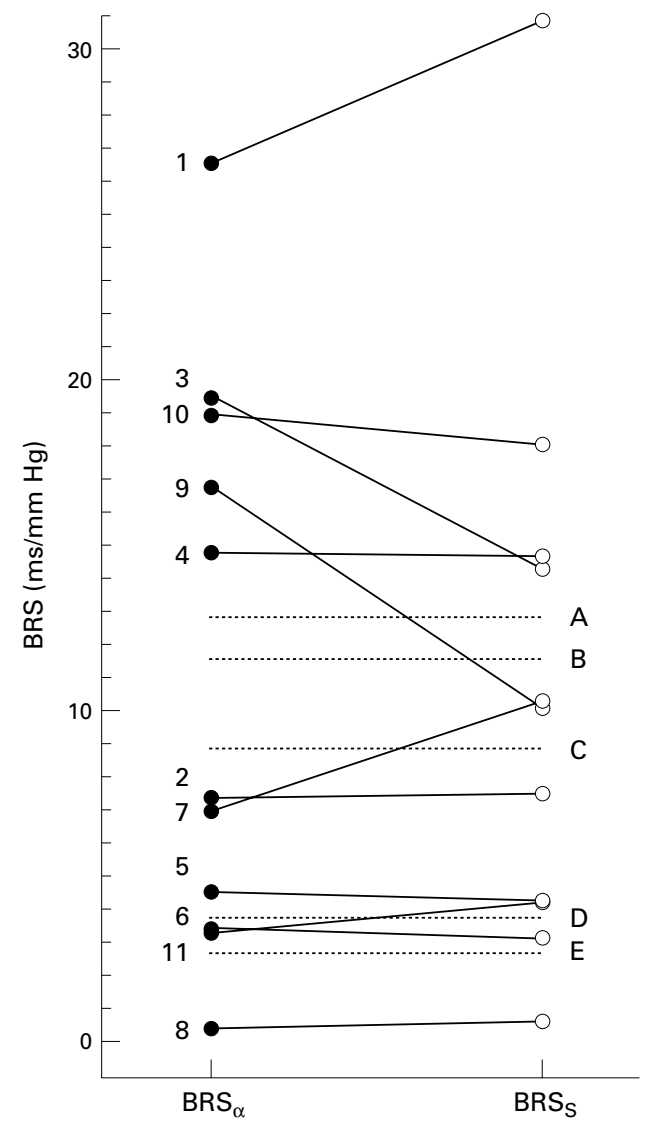

Figure 6 Individual values of baroreflex sensitivity on the heart estimated by the alpha index, $B R S_{a}$ (solid circle), and by the sequence technique, $B R S_{s}$ (open circle), before brain death. Dotted lines are reference values derived from the literature; lines $A$ and $B: B R S_{a}$ and $B R S_{s}$ in healthy volunteers (derived from Kuo et al $)^{8}$ ); line C: $B R S_{s}$ in normotensive subjects during sleeping hours (1200 pm-3 00 am) (derived from Parati et al ${ }^{3}$; lines $D$ and $E: B R S$ and $B R S_{S}$ in vegetative patients (derived from Kuo et al ${ }^{3}$ ).

$\mathrm{Hz}$ (the squared coherence value at this frequency did not differ significantly, being 0.66 and 0.74 before and after brain death, respectively).

ASSESSMENT OF BAROREFLEX FUNCTION

The results obtained by the dynamic assessment of baroreflex sensitivity in the same patient of figure 1 are shown in figure 5 . Values of baroreflex sensitivity as estimated by both the sequence technique and the alpha index dramatically decreased after brain death. In this patient the loss of the baroreflex function was associated with an increase in the hourly number of SBP ramps (an increase in the number of short term SBP spontaneous transients that the sequence technique considers as the probe input to the baroreceptors for evaluating BRS). Baroreflex data averaged over the whole group are reported in table 4 whereas individual BRS values as estimated by the two techniques before brain death are reported in figure 6. As a methodological finding, these data show a substantial agreement between the BRS estimates obtained by the two techniques. This finding is supported by a relatively high degree of correlation between each pair of estimates $(r=0.93)$. From the clinical point of view, before brain death our group of patients was 

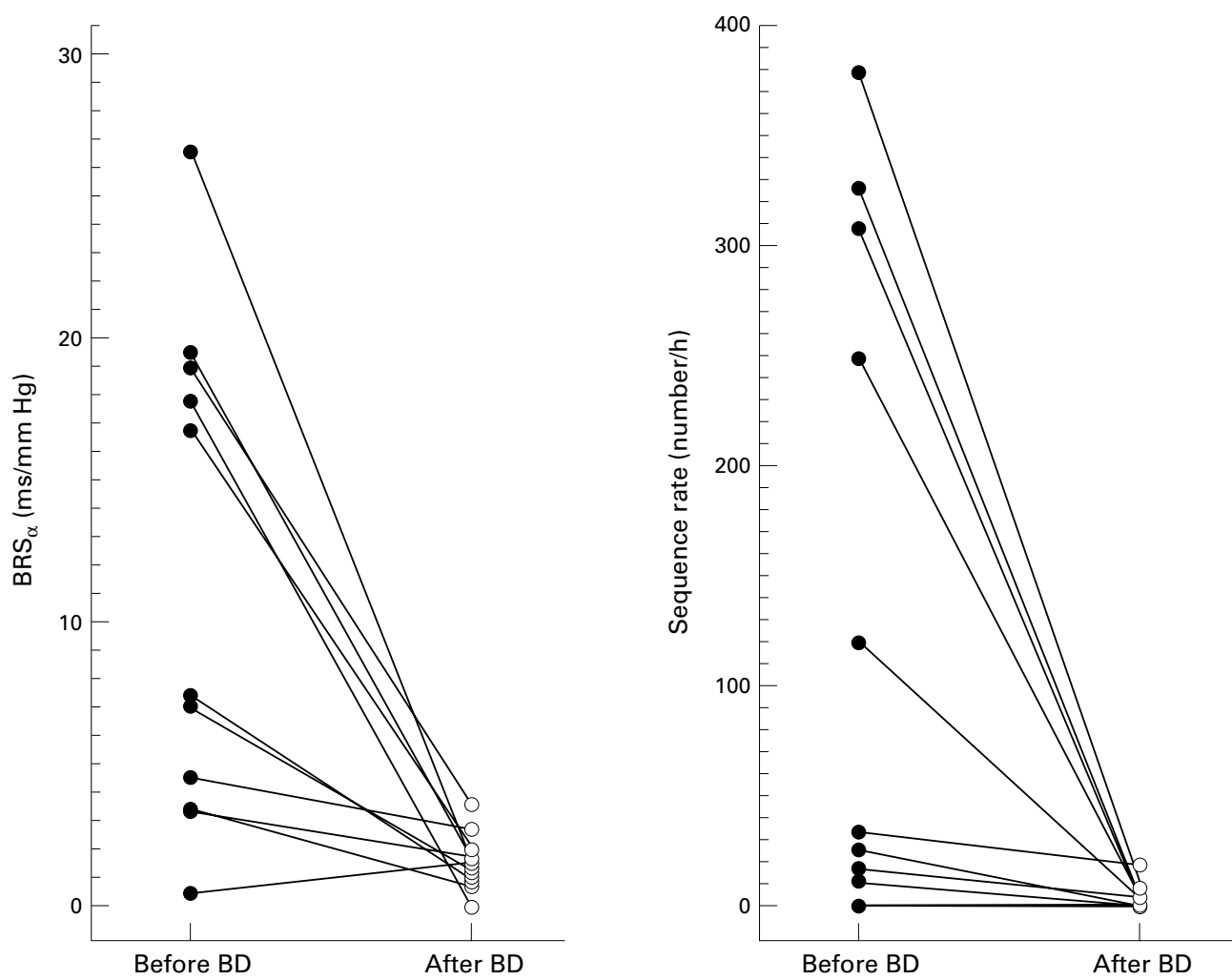

Figure 7 Baroreflex function before (solid circle) and after (open circle) brain death. Left panel: baroreflex sensitivity estimated by the alpha index. Right panel: hourly rate of spontaneous SBP-PI sequences.

characterised by an extremely wide range of individual BRS values, spanning from 0 to 30 $\mathrm{ms} / \mathrm{mm} \mathrm{Hg}$, possibly reflecting the variety of their clinical conditions. It is worth noting, however, that when the individual estimates were averaged over the whole group (table 4), the average BRS value was not too far from the average values found in healthy subjects in the awake condition (around $12 \mathrm{~ms} / \mathrm{mm} \mathrm{Hg}$ ), ${ }^{8}$ or during sleep (around $9 \mathrm{~ms} / \mathrm{mm} \mathrm{Hg}$ ). ${ }^{23}$ After brain death, the average estimates of baroreflex sensitivity as obtained by the alpha index (table 4 and figure 7 ) decreased to a mere $15 \%$ of the values found before brain death. The estimate could not even be obtained by the sequence technique because of the very low number of SBP-PI sequences found after brain death. Indeed, the average rate of spontaneous sequences fell from one every 27 seconds (in the before $\mathrm{BD}$ segment) to one every 25 minutes (in the after BD segment), and no sequences at all were found in three patients after brain death. By contrast, after brain death the number of SBP ramps increased, although not significantly, and several hundred short term SBP transients/hour were found (table 4).

Table 4 Baroreflex sensitivity by the spectral $\left(B R S_{2}\right)$ and the sequences $\left(B R S_{s}\right)$ techniques; rate of occurrence of SBP ramps and of SBP-PI spontaneous sequences

\begin{tabular}{|c|c|c|c|}
\hline & Before $B D$ & After BD & $p$ Value \\
\hline $\mathrm{BRS}_{\alpha}(\mathrm{ms} / \mathrm{mm} \mathrm{Hg})$ & $11.2(8.5)$ & $1.7(0.8)$ & $<0.004$ \\
\hline $\mathrm{BRS}_{\mathrm{S}}(\mathrm{ms} / \mathrm{mm} \mathrm{Hg})$ & $10.7(8.6)$ & Not assessed ${ }^{\star}$ & \\
\hline Sequences rate $(\mathrm{n} / \mathrm{h})$ & $134(151)$ & $2.4(3.4)$ & $<0.02$ \\
\hline SBP ramps rate $(\mathrm{n} / \mathrm{h})$ & $634(375)$ & $772(346)$ & NS \\
\hline
\end{tabular}

Values are mean $(\mathrm{SD})$.

*After BD not enough spontaneous SBP-PI sequences were found to estimate BRS (see text).
From the above data it should be noted that one patient (No 8 in table 1 ) showed very low values of PI power at $0.1 \mathrm{~Hz}$, of BRS estimates and of the hourly rate of spontaneous sequences already before brain death and all these indexes did not substantially decrease after brain death. This case will be specifically considered in the discussion.

\section{Discussion}

This is the first study evaluating changes in blood pressure and heart rate spectra and alterations of the baroreflex occurring in the same patient before and after brain death. Our study showed that the death of the brain stem is followed by important changes in the frequency content of blood pressure and heart rate, in their coupling, and in the indexes reflecting the baroreflex function.

\section{BLOOD PRESSURE AND HEART RATE VARIABILITY} Respiratory oscillation

Arterial blood pressure displayed a clear spectral peak at the respiratory frequency and this peak survived brain death. This is not surprising, because it is known that changes in intrathoracic pressure induced by ventilators produce oscillations in arterial blood pressure through mechanical influences on venous return and cardiac output, which are independent of the cardiovascular centres located in the brain stem. Conversely, the loss of the brain stem function resulted in an almost complete abolition of the respiratory peak in the PI spectrum (that is, in an almost complete abolition of the respiratory sinus arrhythmia), only a 
very small peak being spared. Similar results have been found in animals after the surgical opening of the baroreflex loop by sinoaortic denervation. ${ }^{18}$ These findings are thus compatible with the hypothesis that the respiratory arrhythmia is mainly generated by the baroreflex in the attempt to buffer the ventilatory driven oscillation of blood pressure. The minimal residual component of respiratory arrhythmia still present after brain death might be due to the mechanical stretch induced by ventilation on atrial receptors or on the sinus node, as suggested by a similar residual respiratory arrhythmia in patients after heart transplant. ${ }^{24}$ We also noted that before brain death the respiratory peak was surrounded by a zone of pronounced power in the spectra of blood pressure and heart rate, and that this power completely vanished after brain death. Power surrounding the respiratory peak may possibly reflect the gasping activity present in our patients in the before $\mathrm{BD}$ period.

Ten second rhythm

Before brain death SBP, DBP and PI spectra and SBP-PI coherence were characterised by pronounced peaks at $0.1 \mathrm{~Hz}$, which significantly dropped after brain death. An established hypothesis to explain the $0.1 \mathrm{~Hz}$ power is that a " 10 second rhythm" is generated by a resonance phenomenon due to time constants and delays of the baroreflex loop. ${ }^{25}$ Again, this hypothesis is also supported by the results obtained in animals after the surgical opening of the baroreflex. Indeed, the opening of the baroreflex loop in cats caused the disappearance of the $0.1-\mathrm{Hz}$ peak in both blood pressure and heart rate spectra. ${ }^{18} 27$ Thus, the loss of the $0.1-\mathrm{Hz}$ power of SBP, DBP, and PI that we found in our patients after brain death is likely to reflect the opening of the baroreflex loop induced by the deactivation of cardiovascular centres in the brain stem. Our findings are consistent with previous findings reporting that in this frequency band blood pressure and heart rate powers are lower in brain dead patients than in healthy subjects. ${ }^{8}$

Very slow fluctuations

Very low frequency fluctuations of heart rate decreased significantly with brain death. However, this decrease was not homogeneous at frequencies lower than $0.1 \mathrm{~Hz}$, and the slope of the heart rate spectral trend (the " $1 / f$ " component of the spectrum) increased, more than doubling after brain death. This means that after brain death the relative contribution to the overall heart rate variability of the slower heart rate fluctuations increased with respect to the faster fluctuations. It has been reported that the slope of the $1 / f$ component of the heart rate spectrum is much steeper in patients after heart transplant, where it is equal to -2 , than in normal controls, where it is close to $-1 .^{13}$ Thus it is conceivable that the increase in slope we found after brain death results from the cessation of neural modulations on the heart by the cardiovascular centres located in the brain stem. Also the slope of the $1 / f$ trend of blood pressure spectra increased with brain death. At variance from what was found in heart rate, however, the power of the slowest components of blood pressure variability considered in this study did not change significantly. A remarkable power of blood pressure fluctuations at very low frequency has been already found in blood pressure spectra of brain dead patients, and it was ascribed to vasomotor reflex mechanisms modulated by spinal structures. ${ }^{7-9}$ Actually, vegetative reflexes of spinal origin have been found during brain death, ${ }^{28}$ but this explanation can hardly apply for the patients considered in this study. In fact, the time elapsed between the event of brain death and the start of the period of study after brain death is very short-about 1 hour-and in this interval a spinal shock may also exist. ${ }^{15} 16$ The acute transient fall of blood pressure associated with the fall in endogenous catecholamine concentration we found in plasma may confirm the persistence of a central vegetative blockade and a temporary neurogenic spinal shock in our patients also in the after BD period. ${ }^{45}$ Thus we hypothesise that in our brain dead patients an intrinsic vascular contractile mechanism might be present, as that found in isolated arterial vessels, ${ }^{29}$ or that causing low frequency changes in the diameter of the radial artery in humans. ${ }^{30}$ In this context, the short time after the brain death might be a condition in which oscillations in blood pressure of tonic vasomotor origin can emerge as a result of reduced neural control. The loss of the baroreflex buffering action after brain death may facilitate the occurrence of such very low frequency blood pressure oscillations.

\section{BAROREFLEX FUNCTION}

The baroreflex sensitivity was remarkably high before brain death and, on average, close to values reported in normal subjects. ${ }^{83}$ Values, however, were highly dispersed. This may be explained by considering that if higher centres of the brain are lost or damaged but the brain stem is intact, then the baroreceptor reflex might still work efficiently, with a sensitivity even greater than in the healthy condition, given the absence of potential inhibitory influences coming from higher centres. However, it is also possible that in some of our patients the brain stem was also partially and progressively damaged before brain death, and that the cardiovascular centres of the brain stem were already deteriorating. This might justify the wide distribution of sensitivity values we found before brain death. In this context, it may be of interest to consider the case of one patient (No 8) who showed values of baroreflex sensitivity and hourly rate of spontaneous sequences very close to zero even before brain death. This patient was also characterised by very low values of PI power at $0.1 \mathrm{~Hz}$ even before brain death (whereas the blood pressure powers were similar to those found over the whole group, see table 2). In this patient most of the brain stem reflexes used for the diagnosis of brain death were clinically absent during the before $\mathrm{BD}$ period, with the only exception of a gasping breath. Thus, it is likely that in this patient the cardiovascular centres of the brain stem were 
partially damaged, and the autonomic control of the heart was almost completely lost already before brain death, whereas a residual vasomotor control on blood pressure was still present.

With brain death we expect a cessation of the baroreflex function. When the sequence technique is considered, we actually found that the number of spontaneous SBP-PI sequences (namely, the number of patterns used to estimate the baroreflex sensitivity and which are specifically generated by the baroreflex ${ }^{19}$ ) were virtually abolished. For the alpha index, this could be still estimated after brain death because of the minimal residual oscillations still present after brain death but its value was close to zero.

Finally, a methodological aspect concerning the possible interference of catecholamine injection and estimate of baroreflex sensitivity should be clarified. In comatose patients, including our patients, administration of catecholamines is often necessary before and after brain death. ${ }^{58}$ However, the four patients of our group receiving catecholamines before brain death were also those with the lowest values of baroreflex sensitivity (fig 6 and methods). It could be conceived that the catecholamine injection may have interfered with baroreflex function, and thus with the assessment of baroreflex sensitivity. However, anatomical and clinical evidence suggest that these low values of sensitivity are due to the central default, ${ }^{631}$ not to catecholamines. This hypothesis is further supported by the experimental evidence that continuous infusion of catecholamines (adrenaline and noradrenaline) in healthy volunteers does not reduce the sensitivity of the baroreflex. ${ }^{32}$

After brain death, catecholamines were given to all the patients in this study but one (No 10). To exclude the possibility that catecholamine infusion may influence the results obtained after brain death, we considered data from three patients who did not receive catecholamines, but who were excluded from the study because blood pressure recordings were not available before brain death. After brain death, power spectra, coherences and estimates of baroreflex sensitivities in these three patients plus patient No 10 were similar to those obtained in the group of 10 patients who received catecholamines: in particular, average values (SD) of baroreflex sensitivity estimated by the alpha index were $1.3(0.7)$ and $1.9(1.3)$ $\mathrm{ms} / \mathrm{mm} \mathrm{Hg}$ in the groups with and without catecholamines $(\mathrm{p}=0.34$; NS).

\section{Conclusion}

The study of the shift from the state of impending brain death to the complete inactivity of the brain stem by spectral and coherence analysis of blood pressure and heart rate, and by the continuous monitoring of the baroreflex activity, offers interesting new insights into previous data. The maintenance of baroreflex sensitivity and of spectral parameters in an almost normal morphology up to a few hours before brain death is an important finding. Changes occurring with brain stem death are identified as a redistribution toward the lower frequencies of the blood pressure spectra accompanied by a marked fall in the heart rate spectrum, in the coherence between systolic blood pressure and heart rate, and in the indexes of baroreflex spontaneous activity.

Moreover, our study indicates that the continuous monitoring of cardiovascular reflexes may support the diagnosis of brain stem death. It should also be considered that whereas brain stem reflexes traditionally used for the diagnosis of brain death can be checked only periodically, and require a perceptive and experienced physician for reliable testing, the techniques used in this study allow an automatic and continuous monitoring during the whole period of stay in the intensive care unit. Obviously, attention must be exercised while using these indexes. Indeed, it should be considered that before the onset of brain death a dysfunction of the cardiovascular centres of the brain stem may cause alterations of blood pressure and heart rate variabilities and a deactivation of the baroreceptors reflex. Moreover, specific pharmacological therapy or neurological pathologies may alter these changes. Notwithstanding these limitations, our data indicate that the running evaluation of the spectral components of blood pressure and heart rate variabilities and of the baroreflex function might become an additional tool to confirm the diagnosis of brain stem death.

\section{Appendix: details of data analysis}

DATA PREPROCESSING

Beat to beat series of SBP, DBP, and PI contain values which are unevenly sampled because each value is derived by definition from a single heart beat, and the cardiac rhythm changes over time. Unfortunately spectra obtained by using standard techniques of spectral analysis, such as the fast Fourier transform (FFT), may be distorted if the input data are not evenly sampled over time. Thus, to avoid spectral distortions, each beat to beat series was transformed into evenly sampled signals by a two step procedure. Firstly, the samples were interpolated by cubic splines, ${ }^{33}$ to obtain a smooth, continuous line which, in the second step, was resampled evenly at $4 \mathrm{~Hz}$.

\section{SPECTRAL ANALYSIS}

From each resampled series, an array of spectra was computed by the Welch method. ${ }^{17}$ In practice, each series was broken up in 512 second long, $75 \%$ overlapped, data records. Each data record was windowed by the classic Hann window to reduce power leakage error and a power spectrum was computed in each segment by FFT. Finally, a single spectrum was obtained for each patient and for each series by averaging the spectra falling in the before $\mathrm{BD}$ or in the after BD segment.

\section{COHERENCE ANALYSIS}

The squared coherence modulus $|\mathrm{k}(f)|^{2}$ was computed separately in the before $\mathrm{BD}$ and after $\mathrm{BD}$ periods following a scheme similar to the one used for estimating the power spectrum. For spectral analysis, each series was broken up into 512 second, $75 \%$ overlapped, data 
records. Firstly the cross spectrum between SBP and PI was calculated by FFT in each data record, along with SBP and PI spectra. Cross spectrum and spectra of each record were smoothed by a fourth order moving average filter to improve the consistency of the estimates. Then, SBP and PI spectra and cross spectrum were averaged over all the records. Finally, the squared coherence modulus was calculated as the ratio between the squared modulus of the average cross spectrum, and the average SBP and PI spectra. ${ }^{17}$

1 Guidelines for the determination of death. Report of the medical consultants on the diagnosis of death to the President's Commission for the study of ethical problems in medicine and biomedical and behavioral research. $\mathscr{F} A M A$ 1981;246:2184-6.

2 Conference of Royal Colleges and Faculties of the UK. Diagnosis of brain death. BMF 1976;ii:1187-8.

3 Kero P, Antila K, Ylitalo V, et al. Decreased heart variation in decerebration syndrome: quantitative clinical criterion of in decerebration syndrome: quantitative

4 Goldstein B, DeKing D, DeLong DJ, et al. Autonomic cardiovascular state after severe brain injury and brain death in children. Crit Care Med 1993;21:228-33.

5 Goldstein B, Kempski MH, DeKing D, et al. Autonomic control of heart rate after brain injury in children. Crit Care Med 1996;24:234-40.

6 Goldstein B, Toweill D, Lai S, et al. Uncoupling of the autonomic and cardiovascular system in acute brain injury. $A m$ 7 Physiol 1998;275:R1287-92.

7 Kita Y, Ishise J, Yoshita Y, et al. Power spectral analysis of heart rate and arterial blood pressure oscillation in
brain-dead patients. F Auton Nerv Syst $1993 ; 40: 101-7$.

8 Kuo TBJ, Yen HW, Hseu SS, et al. Diminished vasomotor component of systemic arterial pressure signals and component of systemic arterial pressure signals and

9 Novak V, Novak P, de Marchie M, et al. The effect of severe brainstem injury on heart rate and blood pressure brainstem injury on heart rate and
oscillation. Clin Auton Res 1995;5:24-30.

10 Knüttegen D, zur Nieden K, Müller-Gorges MR, et al. Computer aided analysis of heart rate variability in brain death. International fournal of Clinical Monitoring and Com puting 1997;14:37-42.

11 Task force of the European society of cardiology and the North American society of pacing and electrophysiology. Heart rate variability. Standards of measurement, physiological interpretation, and clinical use. Circulation 1996;93: 1043-5.

12 Malliani A, Pagani M, Lombardi F, et al. Cardiovascular neural regulation explored in the frequency domain. Circulation 1991;84:482-92

13 Bigger JT, Steinman RC, Rolnitzky LM, et al. Power law behavior of RR-interval variability in healthy middle-aged persons, patients with recent acute myocardial infarction, persons, patients with recent acute myocardial infarction,
and patients with heart transplants. Circulation 1996;93: 2142-51
14 Conci F, Levati A, Brenna S, et al. Vegetative storm in brain death. Intensive Care Med 1994;40:S62.

15 Crenna P, Conci F, Boselli L. Changes in spinal reflex excitability in brain-dead humans. Electroencephalogr Clin Neurophysiol 1989;73:206-14.

16 Ivan LP. Spinal reflexes in cerebral death. Neurology 1973;23:650-3.

17 Challis RE, Kitney RI. Biomedical signal processing (in four parts). Part 3. The power spectrum and coherence function. Med Biol Eng Comput 1991;29:225-41.

18 Mancia G, Parati G, Castiglioni P, et al. Effects of sino-aortic denervation on frequency-domain estimates of baroreflex sensitivity in conscious cats Am f Physiol 1999;276:H198793.

19 Bertinieri G, Di Rienzo M, Cavallazzi A, et al. Evaluation of baroreceptor reflex by blood pressure monitoring in unanestetized cats. Am F Physiol 1988;254:H377-83.

20 Pagani M, Somers V, Furlan R, et al. Changes in autonomic modulations induced by physical training in mild hypertension, Hypertension 1988;12:600-10.

21 Castiglioni P, Parati G, Omboni S, et al. Broad-band spectral analysis of 24-h continuous finger blood pressure: comparianalysis of 24-h continuous finger blood pressure: compari-

22 Jenkins GM, Watts DG. Spectral analysis and its applications. San Francisco, CA: Holden-Day, 1968

23 Parati G, Di Rienzo M, Bertinieri G, et al. Evaluation of the baroreceptor-heart rate reflex by 24-hour intra-arterial blood pressure monitoring in humans. Hypertension 1988; 12:214-22.

24 Bernardi L, Keller F, Sanders M, et al. Respiratory sinus arrhythmia in the denervated human heart. F Appl Physiol 1989;67:1447-55.

25 deBoer RW, Karemaker JM, Strackee J. Hemodynamic fluctuations and baroreflex sensitivity in humans: a beat-tobeat model. Am f Physiol 1987;253:H680-9.

26 Wesseling KH, Settels JJ. Baromodulation explains shortterm blood pressure variability. In: Orlebeke JF, Mulder G, van Doornen LJP, eds. Psychophysiology of cardiovascular van Doornen LJP, eds. Psychophysiology of

27 Di Rienzo M, Castiglioni P, Parati G, et al. Effects of sino-aortic denervation on spectral characteristics of blood pressure and pulse interval variability: a wide-band approach. Med Biol Eng Comput 1996;34:133-41.

28 Conci F, Procaccio F, Arosio M, et al. Viscero-somatic and viscero-visceral reflexes in brain death. $\mathcal{f}$ Neurol Neurosurg Psychiatry 1986;49:695-8.

29 Johansson B, Bohr DF. Rhythmic activity in smooth muscle from small subcutaneous arteries. Am F Physiol 1966;210: 801-6.

30 Porret CA, Stergiopulos N, Hayoz D, et al. Simultaneous ipsilateral and contralateral measurements of vasomotion in conduit arteries of human upper limbs. Am $\mathcal{F}$ Physiol 1995;269:H1852-8

31 Winchell RJ, Hoyt DB. Analysis of heart-rate variability: a non invasive predictor of death and poor outcome in patients with severe head injury. F Trauma 1997;43:927-33.

32 Tulen JHM, Man In 'T Veld AJ, Van Roon AM, et al. Spectral analysis of hemodynamics during infusions of epinephrine and norepinephrine in men. 7 Appl Physiol 1994;76: 1914-21.

33 Press WH, Teukolsky SA, Vetterling WT, et al. Numerical recipes in Fortran: the art of scientific computing, 2nd ed. New York: Cambridge University Press, 1992. 